\title{
Politikberatung in der schweizerischen Demokratie - Eigenheiten und spezifische Hürden
}

\author{
Martin Lendi
}

\begin{abstract}
Kernaussagen
In der Zeit der EU-seitigen Konzentrations- und Ausweitungstendenzen mag es vermessen sein, unter dem Titel der Politikberatung auf die Schweiz einzuschwenken, steht sie doch gleichsam, inmitten von Europa, abseits der großen Akzente. Bewusst aber dürfte sein, wie sehr die schweizerischen demokratischen, föderativen, freiheitlichen und sozialen Eigenheiten immer wieder überraschende staatspolitische Erkenntnisse vermitteln. Nicht als Rezepte, wohl aber als „Laborbefunde“ von Relevanz, von politischer Tragweite, dürfen sie verstanden werden. Auch mit Blick auf die Erkenntnis, wonach die Demokratie nie zu Ende gedacht ist, weder unter restriktiven noch unter voranschreitenden Gesichtspunkten.
\end{abstract}

\section{Ein schweizerisches Phänomen - abseits der Literatur, abseits von Institutionen}

Nehmen wir es gleich vorweg: Die schweizerische Literatur zur Politikberatung ist schmal (vgl. Lendi, 2004; Lendi, 2005).Einzelne Ökonomen und einige wenige Politologen der neueren Zeit haben sich Gedanken zu diesem Phänomen gemacht. Ihr Interesse galt vor allem den Schattenseiten ihrer Umtriebigkeit und ihrer Ratlosigkeit, den inhärenten Ideologien, den begrenzten Informationsgehalten und den Schwierigkeiten im Umfeld von Ungewissheiten. ${ }^{1}$ In der staatsrechtlichen Literatur fehlt der Begriff der Politikberatung weitestgehend, selbst in den Stichwortverzeichnissen, die an sich Brückenschläge zu verwandten Phänomen bilden müssten (vgl. Haller, 2009; Jagg et al., 2011; Häfelin et al., 2008).

Und nicht minder interessant: Die Zahl professioneller Institutionen der Politikberatung ist auffallend klein. Neben Avenir Suisse - ein Think-Tank der Privatwirtschaft von außerordentlicher Informations- und Überzeugungskraft, gewandt eingebrachter und gekonnt formulierter Publikationen, aber doch gleichsam vorfokussiert auf polit-ökonomische Denkansätze - gibt es nur einige wenige kleinere Institutionen wie das knapp dotierte Liberale Institut in Zürich. ${ }^{2}$ Daneben melden sich gleich mehrere auf öffentliche Beratungsaufträge spezialisierte Planungsbüros, deren Grundhaltung mehr oder weniger bekannt ist, da sie oft bevorzugten Leitideen folgen. Hervorgegangen sind sie weitgehend aus der Zeit der Planungseuphorie zu Beginn der siebziger Jahre. Auf der anderen Seite gibt es seit geraumer Zeit eine größerer Zahl aktiver individueller Politikberater, die sich auch als Politikbeobachter wiederholt mediengewandt äußern - mit Wurzeln einer- seits in der Parteienlandschaft, anderseits in den politischen Wissenschaften und neu in der politischen Geographie. ${ }^{3}$

Gewachsen ist in jüngster Zeit die Zahl der Hochschulinstitute, die sich u.a. über Aufträge aus dem Bereich der Wirtschafts- und Politikberatung alimentieren. Sie konzentrieren sich in der Regel auf Analysen, Wissenserfassung, Erkenntnisgewinne sowie Begründungen im Kontext ihrer Forschungspräferenzen. Eine besondere Rolle spielen die Nationalen Forschungsprogramme, betreut vom Nationalfonds, thematisch mitgeprägt durch Regierung und Verwaltung. Sie sind in der Regel von politischer Relevanz, sachlich herausfordernd, zeitlich eher ausholend und in der Regel interdisziplinär angelegt, sodann wissenschaftlich geführt und begleitet. Sie tragen den Keim der Politikberatung in sich, müssen aber oft erleben, dass sie am Schluss ihrer Arbeiten gezwungen sind, sich mit dem parallel neu gewordenen Stand des Wissens zu konfrontieren, was ihr Beratungsgewicht erheblich mindern kann.

Der Kontrast zu diesen spezifischen Akteuren ist die Tagesaktualität: Unsummen an Geldmitteln geben die Verwaltungen - vor allem die Bundesverwaltung - für Gutachten, Expertisen und Forschungsarbeiten (eher monothematischer Art) direkt adressiert an einzelne Akteure aus. Deren Arbeiten sind in der Regel als echte, Quasi- oder Alibi-Politikberatung“ mehr oder weniger von Relevanz, mehr oder weniger von präjudizieller Bedeutung. Ob sie einen kritischen Beitrag an die laufende Meinungsbildung in den Verwaltungen, Regierungen und Parlamenten leisten, ist mindestens teilweise als offen $\mathrm{zu}$ betrachten, da nicht ausgeschlossen werden kann, dass Mandate oft zur Unterstützung vorgefasster Meinungen vergeben werden oder Aktivismus resp. Hinhalten zu rechtfertigen haben. Als relativ sorgfältig darf die eigentliche Ressortforschung bezeichnet werden, soweit sie als echte Forschung instradiert wird. Sie wirkt aus sich heraus politikberatend. Wie dem auch sei, die Politikberatung ist - mit andern Worten -

\footnotetext{
1 Zu den wichtigsten Autoren zählen Bruno S. Frey, René L. Frey, Gerhard Kirchgässner, Wolf Linder, Dietrich Ruloff und Hans Würgler. Rechtswissenschaftler sind weniger ausgeprägt präsent, obwohl einige als Parlamentsabgeordnete am Politikgeschehen mitwirkten, etwa Jean François Aubert, Richard Bäumlin, Riccardo Jagmetti, René Rhinow, Ulrich Zimmerli; außerdem Kurt Eichenberger, der als Professor ein eng vernetzter Berater und Gutachter des Bundesrates war.

2 Think-Tanks sind nicht prototypisch für die schweizerische Politikberatung. Diese funktioniert über Angehörige aus dem Hochschulbereich, aus dem Verbandswesen und über private Büros. Eine große Rolle spielt auch das Gutachterwesen, beispielsweise in juristischen Belangen.

3 Vor allem die Universität Bern förderte zahlreichen Nachwuchs für die Politikberatung, inspiriert u.a. von Wolf Linder, siehe bspw. seine Liste privatwirtschaftlicher und öffentlicher Think-Tanks in der Schweiz (Steffen \& Linder, 2006). Ob diese alle als unabhängige, kontinuierlich wirkende Politikberater fungieren, die über ein eigenes, unterlegtes Politikwissen verfügen, darf kritisch gefragt werden.
} 
präsent, wenn auch nicht fest verankert, gar institutionalisiert. Gegenwärtig ist sie allemal.

\section{Politikberatung - vom Abstraktum zu konkreten Ausformungen?}

Was Politikberatung ist, wie sie sich versteht, wie sie Einfluss nimmt und gewinnt, ob und wie sie beachtet resp. gar befolgt wird, das kann als abstraktes Thema angegangen werden. Und so fehlt es nicht an Abhandlungen, die sie vorauseilend begrifflich bestimmen, beispielsweise als an Behörden adressiertes Beantworten offener sachlich-fachlicher Fragen, in Front nachfolgernder politischer Wertungen und angelegt auf Dis$\operatorname{tanz}$ zu den eigentlichen politischen Entscheidungsprozessen, also wissenschaftlich angedacht und gehalten auf Distanz zur politischen Bewertung, jedenfalls fern versuchter politischer Einflussnahme. Im Wesentlichen geht es wohl um den Wissenstransfer aus der Wissenschaft in die Politik, unter pointierter Abgrenzung zu jenen Ratschlägen, die politische Wertungen zu beeinflussen trachten, allenfalls sogar im besonderen Interesse der Intervenierenden.

Ob die Definition alle Variationen der Politikberatung abdeckt und den mitschwingenden Nuancierungen gerecht wird, darf und muss gefragt werden. Sei es funktional, sei es theoretisch, sei es bezogen auf die Einbindung in die politische Kultur und/oder die Alltagswirklichkeit politischen Geschehens. Wie insbesondere das üblicherweise verwendete Merkmal der „Wissenschaftlichkeit“ zu verstehen ist, bleibt heikel. Geht es um einen Hinweis auf das angebrachte, vorausgesetzte Verwenden wissenschaftlicher Methoden? Steht eine materielle Beschränkung auf den Wissenstransfer aus der Wissenschaft in die Politik in Frage - unter Ausschluss jener Politikberatung, die nicht institutionell in ihr verankert ist? Mindestens in der Schweiz ist, für den gesamten Politikbereich, eine Grundhaltung auszumachen, die ein Hervorheben des Wissenschaftlichen vermeidet, auch wenn der Kontakt zur Wissenschaft in der Realität lebhaft gesucht wird. ${ }^{4}$

Was immer unter Politikberatung verstanden wird. sie bleibt beratend. Sie beansprucht keine Entscheidungskompetenzen, da sie von einer rechtlich/politisch gefügten Kompetenzordnung und von zu respektierenden, rechtlich strukturierten Entscheidungsprozessen ausgeht, auch wenn sie oft genug zu verstehen gibt, ihre sachlichen Aussagen kämen an das Zweckmäßige, Gerechte, Gebotene und Verbindliche heran, also an die Maßstäbe des staatlichen Handelns (Lendi, 2012). Dies sind gleichsam die Kernelemente des Politischen, deren erstverantwortliche Träger nicht die Berater sind, sondern die Organe, die Repräsentanten der Politik.

Wendet man sich den Funktions-, Sinngebungs- und Erfahrungswelten mit der Politikberatung in einem konkreten Staat zu, nimmt das Phänomen konkretere Züge an. Selbst in europäischen Staaten mit einer über alles gesehen gemeinsamen Wertekultur und einem hochgehaltenen Demokratieverständnis schleichen sich nationale oder gar regionale Differenzierungen ein. Die Politikberatung wird sich in einer Präsidialdemokratie de facto anders ausnehmen als in einer parlamen- tarischen mit Institutionen von Regierung bis Verwaltung und vor allem hinsichtlich der Kommissionsarbeiten im Parlament. Hier kann Politikberatung für die Parlamentarier direkt erlebbar werden.

\section{Sonderfall Schweiz?}

er „Sonderfall der schweizerischen Demokratie“ liegt nicht auf der Hand. Sie weist nebst Elementen der parlamentarischen Demokratie Instrumente der direkten auf, z.B. Referenden und Initiativen, welche die Politikberatung, hier noch als These eingebracht, in einem etwas anderen Licht erscheinen lassen.

Die Politikberatung erreicht ihren Höhepunkt nicht im Vortrag beim zuständigen Minister oder in einer parlamentarischen Kommission, so bedeutsam sie auch sein mögen. Die Politikberater sehen sich vielmehr häufig vor die offene Tür hin zu einer Volksabstimmung gestellt. Was dies für sie und die Politikberatung genau bedeutet, muss bedacht werden. Offenkundig ist, dass sie im Sinne des verbreiteten Wissenstransfers an die üblichen staatliche Organe in vielen Fällen das Volk als letztlich entscheidendes Staatsorgan nicht oder nur sehr indirekt erreicht. Sie endet in der Regel in den parlamentarischen Vorphasen der Gesetzgebung und des Umganges mit Referenden sowie Initiativen.

Die Beschlüsse der Parlamente des Bundes, der Kantone und größerer Gemeinden stehen in der Demokratie schweizerischer Prägung „ex constitutione aut ex lege“ unter dem Vorbehalt obligatorischer oder fakultativer Referendumsmöglichkeiten, mithin von Volksabstimmungen. Auch Initiativen, aus dem Volk heraus, können durch abschließendes Volksvotum geltendes Verfassungs- oder Gesetzesrecht werden, sogar gegen den Willen von Parlament, Regierung und Verwaltung. Die Beratungen in den zuständigen Organen stehen somit unter dem nicht seltenen Vorbehalt konstitutiver Volksabstimmungen. ${ }^{5}$

Selbstredend sind die rechtlichen Anforderungen differenziert und also nicht so einfach angelegt, wie diese kurze Zutrittsformulierung vermuten lässt. Das Prinzip aber ist erhellt. Politische Rechte der Bürgerinnen und Bürger sind unter verfassungsrechtlichen Vorgaben involviert, konkret ihre Stimmrechte zu Erlassen und Vorhaben, neben den Wahlrechten. So hat sich die Politikberatung, wo und in welchen Phasen der Entscheidungsprozeduren auch immer, letztlich auf das verbindliche Bürgervotum auszurichten. Ob dieses funktional im Referendumsbereich als „Veto“ verstanden wird, ob für die Politikwissenschaft das Volk als Träger der

4 Ich habe ein materielles Verständnis bevorzugt und auf eher formale Begriffsmerkmale bewusst verzichtet (Lendi 2005). Im Vernehmlassungsverfahren zu Erlassen und Vorhaben werden keine Anforderungen zur Wissenschaftlichkeit gestellt. Dieses Begriffsmerkmal scheint jedoch als Abgrenzungen zu nicht substanzieller, eher willkürlicher, interessengebundener „Politikberatung“ veranlasst, nur ist die Wissenschaft nicht davor gefeit, die strengen Pfade hoher Wissenschaftlichkeit bisweilen zu verlassen.

5 Siehe dazu für die Ebene des Bundes Art. $138 \mathrm{ff}$. BV. Für die föderative Ebene sind die 26 kantonalen Verfassungen zu konsultieren. 
„Opposition“ gedeutet wird, ist für diesen Zusammenhang nicht entscheidend. Maßgebend ist, dass das Volk aus der Verfassung heraus ein vorausgesetztes Organ ist.

Dies ist die spezifische Dimension der Politikberatung in der schweizerischen Demokratie. Sicherlich ist diese Flanke während der verwaltungsnahen Vorphasen noch wenig spürbar. Schon gar nicht bei abschließender Zuständigkeit der Verwaltung, der Regierung oder des Parlamentes. Je näher aber Entscheidungsprozesse an die Endphase vor einer Volksabstimmung heranrücken, desto manifester wird die offene Seite bereits für die Regierung, erst recht für das Parlament und seine Kommissionen, oft gar virulent. Verwunderlich wäre es, wenn die Politikberatung auf Distanz gehen könnte.

Die Eigentümlichkeiten haben zu einem Sonderinstrument geführt. Die Bundesverfassung der Schweizerischen Eidgenossenschaft (BV) sieht für die Vorbereitung wichtiger Erlasse und anderer Vorhaben von großer Tragweite das „Vernehmlassungsverfahren“ vor. Dieses lädt die Kantone, die politischen Parteien und die interessierten Kreise zur Stellungnahme ein - gleichsam eine institutionalisierte, weitgehend offene „Politikberatung“. Die eingegangenen Meinungsäußerungen werden ausgewertet. Darüber wird in den Berichten (Botschaften) der Regierung zu ihren Vorlagen, gerichtet an das Parlament, berichtet. ${ }^{6}$ Die Vernehmlassung dient dem Erfassen der vorherrschenden Interessen, dem Ermitteln der Notwendigkeit und Verhältnismäßigkeit von Regelungen, dem Wecken des Problembewusstseins in breiten Kreisen und letztlich der Ausarbeitung von Vorlagen, die vor dem Referendum bestehen könnten. Es ist ein Gefäß und zugleich ein Aktionsfeld für Politikberater, sich selbst einzubringen oder sich in den Dienst der sich Äußernden zu stellen. Auf spezifische Nuancierungen von Referendum und sachlich-politische Akzente setzendem Initiativerecht wird nun mit Blick auf ihre Relevanz für das Verständnis der Politikberatung eingegangen.

\section{Die Vorwirkung des Referendums}

Eine der wichtigsten Komponenten des Verständnisses der schweizerischen Politik hat mit dem Verfassungs- und Gesetzesreferendum, auf kantonaler Ebene sogar zusätzlich mit dem Finanzreferendum, zu tun. ${ }^{7}$ Dieses handelt von der entscheidungsprozeduralen „Nachhut“ zum Parlament, die aber nicht als gewillkürtes Anhängsel fungiert, sondern von Rechtswegen konstitutiv wirkt. Außerdem zeitigen das vorbehaltene obligatorische und fakultative Referendum Vorwirkungen auf die Entscheidungsetappen, bestimmend auch für Politikberatung bis in Parlament, Regierung und sogar Verwaltung hinein, weil vorhersehbare resp. mögliche Volksabstimmungen bedacht sein wollen. ${ }^{8}$

Wer mit Verfassungs- und Gesetzgebungsbelangen betraut ist, hat die Akzeptanz eines Erlasses oder eines Vorhabens nicht nur bei den staatlichen Organen im engeren Sinn zu bedenken, sondern eben auch im Volk. Ohne dessen aktive Zustimmung oder den gewollten Verzicht auf ein fakultatives Referendum können die getroffenen Beschlüsse nicht in Kraft treten. Es handelt sich nach schweizerischem Recht gerade nicht um ein konsultatives oder gar um ein ad hoc inszeniertes, sondern um ein durch das Recht vorgesehenes obligatorisches oder ein fakultatives, d.h. auf Begehren eines Quorums der Bürgerschaft stattfindendes Referendum. ${ }^{9}$ Das Ergebnis ist verbindlich und rechtlich konstitutiv. Die Referendumsmöglichkeiten sind zudem von erheblicher politischer Bedeutung, etwa durch ihre Vorwirkung auf das Parlament, selbst in jenem nicht seltenen Fall eines Fakultativen, das unter Umständen nicht ergriffen wird.

Übertragen auf die Politikberatung bedeutet dies, dass die Gesprächsdimension zwischen dem Auftraggeber zur Politikberatung und dem Mandatsträger resp. zwischen dem sich spontan einbringenden Beratern und dem adressierten staatlichen Organ erweitert wird um das schwer fassbare Volk als Staatsorgan, das sich an einem noch nicht präzise festgelegten Zeitpunkt äußern wird - oder im Falle des fakultativen Referendums vielleicht auch darauf verzichtet. Vereinfacht ausgedrückt, wer immer sich in der Schweiz politikberatend äußert, der muss sich Rechenschaft geben, dass es neben dem direkten Gesprächspartner, den man berät, möglichweise am Ende des Entscheidungsprozesses ein Volksvotum gibt oder geben könnte - unter dem rechtlichen Vorbehalt abschließender Zuständigkeiten des Parlaments oder der Regierung.

Unter diesen Umständen muss die Politikberatung in der Referendumsdemokratie immer auch auf den potentiellen Volkswillen schauen resp. am direkten Adressaten vorbei auf das Volk als Staatsorgan verweisen. Dabei sind die geltenden Maßstäbe des Zweckmäßigen, des Gerechten, des Gebotenen und des Verbindlichen zu beachten. So bleibt alles in allem Zurückhaltung gegenüber den politischen Entscheidungsprozessen zu wahren, zumal die Politik - nicht die Berater - ihre Entscheidungskompetenzen zu verantworten hat. Dennoch können die Wirkungen und Auswirkungen eines Erlasses oder eines Vorhabens auf die breite Öffentlichkeit nicht einfach außer Acht gelassen werden. Sie sind vielmehr vorwegnehmend durch die beteiligten Organe eingehend zu bedenken und schon ist die Politikberatung direkt oder indirekt auch in diese Dimension einbezogen, nicht als selbständiges Votum, aber doch in der Sache beratend, beispielsweise wie sich eine sachliche Regelung unter Gesichtspunkten der Akzeptanz und

6 Maßgebend sind Art. 147 BV sowie das Bundesgesetz über das Vernehmlassungsverfahren vom 18. März 2005 samt zugehöriger Verordnung. Die besondere Funktion dieses Angebotes besteht in der Rücksichtnahme auf die Möglichkeit eines Referendums, s.u.

7 Zum Referendum siehe Art. 140/141 BV. Das obligatorische Referendum gilt für Änderungen des Bundesverfassung, den Beitritt zu internationalen Organisationen für kollektive Sicherheit und zu supranationalen Gemeinschaften usw. Das fakultative Referendum kann von 50.000 Stimmberechtigten ergriffen werden und betrifft vorweg Bundesgesetze, aber auch gewisse völkerrechtliche Verträge usw.

8 Das Problem der Vorwirkung berührt eine der zentralen Komponenten zum Verständnis der schweizerischen Politik, siehe hierzu Neidhart (1970) sowie neuerdings Linder (2008).

9 Vgl.zu konsultativen Abstimmungen Zaccaria (1994). Bei der Kernfrage nach Ad-hoc-Volksbefragungen ist zu bedenken, dass diese in der Regel „unechte“ Volksbefragungen sind, weil weder Rechtmäßigkeit noch Verbindlichkeit hinreichend anhand aussagekräftiger gesetzlicher Grundlagen überprüft werden können. 
der anzustrebenden Zustimmung des Volks resp. der Stimmberechtigten ausnehmen soll, wobei das Sachliche/Fachliche für die Politikberater zu dominieren hat. ${ }^{10}$

Diese Feststellung wirkt sich auf das schweizerische Verständnis der Politikberatung aus. Die Berater, die selbst aktive Bürgerinnen und Bürger sind, tönen in ihren Aussagen, bei aller Zurückhaltung, gleichsam Gedanken an, die über das, was sachlich zweckmäßig erscheint, hinaus reichen, die implizieren, was vor dem Volk und für die Stimmberechtigten als zweckmäßig, gerecht, ethisch geboten und verbindlich erscheint. Die Beschreibung der Politikberatung als wertneutrale, wissenschaftliche Sach- und Erkenntnisvermittlung kann so, mindestens für die Schweiz, nicht völlig zutreffen; verbunden mit dem Vorteil, dass gute Politikberatung die Legitimierungsaspekte nicht übergehen kann und darf. Diese sind für die Sache, die Politik, deren Glaubwürdigkeit und erst recht für die Beständigkeit des geäußerten demokratischen Willens von Bedeutung.

\section{Das Initiativrecht als heikles Feld der Politikberatung - Rückbezüge zum Unterschwelligen}

Für die Politikberatung wesentlich heikler sind Volksinitiativen. Auf Bundesebene geht es um die sog. Verfassungsinitiative. ${ }^{11}$ Sie zielt im politisch relevanten Kern auf eine Teilrevision der Verfassung, die sogar als ausgearbeiteter Entwurf eingereicht werden kann. Sie wird, sofern die verfassungsrechtlichen Voraussetzungen erfüllt sind, ex constitutione dem Volk und Ständen (Kantone) zur Abstimmung unterbreitet. ${ }^{12}$ Das Parlament empfiehlt sie zur Annahme oder Ablehnung, es kann der Initiative einen direkten oder indirekten Gegenvorschlag (Gegenentwurf) ${ }^{13}$ gegenüberstellen. So besehen beschränkt sich die Politikberatung im Wesentlichen auf die Auseinandersetzung zu Gründen der Annahme oder Verwerfung resp. zum Problembereich der Vorlage eines direkten oder indirekten Gegenvorschlags. ${ }^{14}$

Nicht im Zentrum für die Politikberatung stehen das Angehen, das Formulieren, das Ergreifen einer Verfassungsinitiative. Diese Schritte werden in der Regel im inneren Kreis eines Initiativkomitees unternommen, es sei denn, dieses trete von sich aus mit den Anliegen an die Öffentlichkeit, was vor allem dann der Fall ist, wenn Parteien oder Verbände eine Initiative ankündigen und lancieren. Aber selbst in einem solchen Fall stehen die staatlichen Organe vor der Gegebenheit, dass eine Verfassungsinitiative gleichsam ohne Politikberatung auskommt, bis Verwaltung, Regierung und Parlament von der Schlüsselfrage nach der Empfehlung einer Annahme, Ablehnung oder der Ausarbeitung eines Gegenvorschlages eingeholt werden. Immer aber ist die rechtsgültig zustande und formell/ materiell rechtmäßige Initiative ein vorgegebenes Faktum, das dem Verfassungsgesetzgeber zu unterbreiten ist, es sei denn, die Initiative werde vom Initiativkomitee zurückgezogen.

Ein Kernproblem ergibt sich für die Politikberatung aus einem merkwürdigen Umstand. Volksinitiativen - unbesehen des nüchternen Anliegens eine Teilrevision der Verfassung erfüllen oft, politisch gewichtet, eine Ventilfunktion aus dem
Unterschwelligen aus. ${ }^{15}$ Sie artikulieren zwar politisch relevante Problemstellungen, doch sind sie nicht selten umgeben, angereichert, beeinflusst von unbestimmten, aufgestauten, unterschwellig gärenden Elementen des öffentlichen, politischen Unbehagens, welche die Stufe der politischen Traktandenliste noch nicht erlangt resp. geschafft haben. Initiativen sprechen also häufig das Fluidum des Unbewussten, des Unterbewusstseins der Öffentlichkeit an, auch wenn sie - scheinbar widersprüchlich - nüchterne Sätze des zu novellierenden Verfassungsrechts zum Gegenstand haben.

Die Politikberatung zu solchen Initiativen mit Ventilfunktionen kann sich nicht darauf beschränken, die Komponenten des Zweckmäßigen, des Gerechten, des Gebotenen und des Verbindlichen zur Sprache zu bringen. Sie hat das Hintergründige, das Unterschwellige zu ergründen, bestmöglich zu verstehen. Allein schon deshalb, weil in der anstehenden Volksabstimmung das Unterschwellige neben dem Sachlichen aufflackernd präsent sein wird, übrigens in den aktuellen Ausprägungen im Zeitpunkt der Volksabstimmung. Die Politikberatung hat sich in der Regel nicht direkt zum Unterschwelligen zu äußern, doch kann sie nicht daran vorbei sehen, weil selbst die für sie dominierende sachliche Komponente vom Unterschwelligen berührt wird oder werden könnte.

\section{Fallbeispiele}

Die attraktiven Fallbeispiele bewegen sich weitgehend im Kontext der Verfassungsinitiativen. Die Stichworte des Minarett-Verbots, der Unverjährbarkeit der Strafverfolgung und der Strafe bei sexuellen und pornografischen Straftaten an

10 Dies ergibt unter drei Gesichtspunkten Sinn: a) Augenmaß für eine tragbare, sinnvolle Regelung, sachlich und rechtsethisch; b) Blick auf die Akzeptierbarkeit und die Zustimmung des Volkes; c) gerichtet auf die zu gewinnende Legalität und Legitimität staatlichen Handelns.

11 Siehe dazu Art. $138 \mathrm{ff}$. BV, aber auch Art. $192 \mathrm{ff}$. BV. Die geltende Bundesverfassung kennt als Volksinitiative nur die Verfassungsinitiative, nicht die Gesetzesinitiative. Auf kantonaler Ebene sind beide Instrumente bekannt. Diese Grundordnung ist nicht unproblematisch, weil für nicht wenige Anliegen die Gesetzesinitiative rechtlich genügen würde. Ein heikles Thema bilden die „Schranken“, die dem Initiativrecht gesetzt sind. Die Verfassung erwähnt für die Volksinitiative auf Teilrevision die Einheit der Form, die Einheit der Materie sowie das zwingende Völkerrecht, das eher restriktiv auf Elementares reduziert verstanden wird. Außerdem gäbe es noch die Volksinitiative auf Totalrevision der Bundesverfassung, ein politisch kaum relevanter Fall. Die Initiative zur Teilrevision spielt demgegenüber eine politisch große Rolle, zahlmäßig und als politischer Stimulans. Sie kann von 100.000 Stimmberechtigten ergriffen werden.

12 Art. 139 Abs. 5 BV.

13 Auf Bundesebene zielt der direkte Gegenentwurf gleich der Initiative auf die Stufe der Verfassung, der indirekte soll auf Gesetzesebene oder in anderer Beschlussform ergehen.

14 Regierung und Parlament prüfen, ob eine Initiative gültig ist; das Parlament entscheidet, nicht ein Gericht (Art. 139 Abs. 3 BV). Die verfassungsrechtlichen Gültigkeitsvoraussetzungen sind restriktiv gefasst.

15 Die schweizerische Rechtsliteratur, aber auch die politikwissenschaftliche, befasst sich kaum mit dem Unterschwelligen (vgl. Lendi 2009), welches da und dort im Verdacht steht, Aufhänger für populistische Parteien und Parteiprogramme zu sein. Diese Sicht genügt nicht, so begründet sie sein mag. In der Demokratie mit dem Volk als Staatsorgan spielen auch dessen Wahrnehmungen eine wichtige Rolle und können im Zusammenhang mit Volksabstimmungen relevant werden, wenn sie Aufgestautes direkt oder indirekt berühren; kanalisiert auf Wahlen ist das Phänomen bekannt. 
Kindern vor der Pubertät usw. tönen an, wie sehr sich Sachliches mit Politischem aus dem Unterschwelligen vermengen kann. ${ }^{16}$ Diese Durchmischung belastet oder überfordert möglicherweise - die Politikberatung; sie kommt auf alle Fälle über das rein Sachliche nicht voll zum Tragen. Im Referendumsbereich, wie er oben gleichsam an erster Stelle angesprochen wurde, ist der Vorlaufprozess ein wesentlich differenzierterer. Dieser lässt Politikberatung aufblühen, er schafft Zeit und Raum für die Politikberatung. Dies soll an je einem Fallbeispiel näher erläutert werden.

\subsection{Entstehung des Bundesgesetzes über die Raumplanung und seine laufenden Revisionen - Referendumssituation}

Im Jahre 1969 erging der Verfassungsartikel über die Raumplanung, gestützt auf ein obligatorisches Referendum - mit Volks- und Stände(Kantons-)mehr. ${ }^{17}$ Verwaltung, Bundesrat, Parlament machten sich unverzüglich an die Arbeit. Die Erstarbeiten wurden einer Expertenkommission anvertraut.

Als erstes erging, nach durchgeführten Expertenanhörungen durch die Expertenkommission, ein Bundesbeschluss über dringliche Maßnahmen auf dem Gebiet der Raumplanung, der das Ausscheiden provisorischer Schutzgebiete vorsah, ohne dass das fakultative Referendum ergriffen worden wäre. Der bald schon von der Expertenkommission vorgeschlagene Gesetzesentwurf wurde, nach einem breiten Vernehmlassungsverfahren bei Parteien, Verbänden, Kantonen usw., von der Regierung verabschiedet und vom Parlament, bereichert mit bodenrechtlichen Anliegen und einem volkswirtschaftlichen Ausgleich zugunsten der Berggebiete, nach langwierigen Beratungen am 4. Oktober 1974 erlassen. In der Folge wurde aber das (fakultative) Referendum ergriffen. In der Volksabstimmung vom 13. Juni 1976 wurde das Gesetz abgelehnt, wohl vor allem der relativ weitgehenden bodenrechtlichen Einschränkungen wegen.

Unverzüglich kam es zu einer neuen, kleinere Expertenkommission, die unter Auswertung der Ablehnungsgründe und nach erneuten Anhörungen einen neuen Entwurf vorlegte, der nach durchgeführter Vernehmlassung von der Regierung und vom Parlament am 22. Juni 1979 sach- und zeitgerecht verabschiedet wurde. Das fakultative Referendum wurde bei diesem zweiten Anlauf nicht ergriffen. Der Gesetzgeber hat sich ein dynamischeres Planungsverständnis zu Eigen gemacht und die bodenrechtlichen Beschränkungen des Eigentums reduziert. Das neue Gesetz trat am 1. Januar 1980 in Kraft. Es wurde seither mehrfach revidiert, zuletzt gleichsam als indirekter Gegenvorschlag zur sog. Landschaftsinitiative, welche die bestehenden Bauzonen einfrieren wollte, neu angereichert mit verschärften Bestimmungen planerischer und bodenrechtlicher Art - ohne dass je das (fakultative) Referendum bis dato ergriffen worden wäre.

An diesem Beispiel fallen drei Grundelemente auf: Neben der ordentlichen Funktionserfüllungen der Verwaltung, der Regierung und des Parlamentes spielte die Politikberatung eine zentrale, große Rolle, sei es im Rahmen gewichtiger Expertenkommissionen, sei es aufgrund gezielter Experten- aufträge, sei es im Rahmen der parlamentarischen Kommissionsarbeit. Zweitens wurde das Instrument des Vernehmlassungsverfahrens beim ersten und zweiten Gesetz mit Akribie ausgewertet, um Schwachpunkte zu vermeiden und um die parlamentarischen Beratungen gehörig bereichern zu können. Neben den fachorientierten Politikberatern kamen auch Berührte, Betroffene, Interessierte und Engagierte zum Wort von den Parteien und Verbänden über Private bis zu den Kantonen und Gemeinden. Drittens: Die Gesetzgebung wurde politisch gewichtet - stets mit Blick auf das Referendum auf Akzeptanz hin geeicht, allein schon um die Zustimmung des Volks doch noch zu erlangen. ${ }^{18}$ Nur, beim Gesetz von 1974, das abgelehnt worden ist, war der Wissensstand rund um die Raumplanung noch kaum gefestigt, selbst bei den Politikberatern. Es gab damals nur wenige, die „sattelfest“ zu agieren wussten. Anders beim zweiten Gesetz, dem heute noch geltenden RPG. Hier vermochten die Politikberater bereits wesentlich kompetenter aufzutreten. Dennoch, auch bei unterschiedlichem Reifegrad hatten die Politikberater neben dem Zweckmäßigen, dem Gerechten, dem Gebotenen und dem Verbindlichen stets die erforderliche Zustimmung des Volkes im Blickwinkel, sachlich zwingend, politisch angezeigt. ${ }^{19}$

\subsection{Zweitwohnungs-Initiative - Initiativsituation}

Das Problem der gesellschaftlichen, räumlichen und landschaftlichen Belastung durch den Zweitwohnungsbau, ausgeprägt erfahrbar in den Touristenregionen der Berggebiete, aber auch bis in die Städte hinein, ist nicht ein neues Problem. Leider wurde es durch die Kantone, die für die Raumplanung zuständig wären, nicht wirksam genug angegangen. Darüber entstand weitherum Unbehagen. Dies führte zu aufgestauten Vorwürfen im öffentlichen Unterbewusstsein, auch deshalb weil die offizielle Politik die „Missstände“ überging. Die

16 Seit dem Erlass der Bundesverfassung vom 18. April 1999 sind gleich mehrere Volksinitiativen als Verfassungsinitiativen in der Volksabstimmung angenommen worden, obwohl sie zur Ablehnung empfohlen waren: Das Unterschwellige machte sich Luft, so in den Beispielen Minarett-Initiative (Art. 72 Abs. 3 BV), Verwahrung bis zum Lebensende für gewisse Sexual-und Gewalttäter (Art. 123a BV), Unverjährbarkeit der Strafverfolgung und der Strafe bei sexuellen Straftagen (Art. 123 b BV); neuerdings die Zweitwohnungen (Art. 75 b BV); schon vor der Verfassungsrevision z.B. die Initiative zum Schutz des Alpengebietes vor Transitverkehr (Art. 84 BV).

17 Zur Geschichte der schweizerischen Raumplanung siehe Lendi 2006 zu rechtlichen Grundlagen Ruch (2010) und Lendi (1997; 2008). Besonderes Kennzeichen ist, wie eine öffentliche Aufgabe, die Einschränkungen für Grundeigentümer sowie Kantone und Gemeinden mit sich bringt, einen relativ hohen politischen Stellenwert erreichen kann - mitten im unvermeidbaren Wandel des Planungsverständnisses und der Planungsfunktionen.

18 Eine Eigenheit des schweizerischen Rechtsetzungsverfahren ist, dass die Stimmberechtigten gleich zweimal zum Zug kommen: obligatorisch auf Verfassungsebene bezüglich Kompetenz und materiellem Auftrag an den Bund, sowie auf Gesetzesstufe zur gleichen Materie mit dem fakultativen Referendum, wo es um das Was und das Maß wie auch um das Wie geht.

19 Eine zweite Ablehnung wäre sachlich problematisch gewesen, allerdings mussten Abstriche in Kauf genommen werden. Sie betrafen u.a. bodenrechtliche Aspekte, die in der gegenwärtig anschwellenden Diskussion um Revisionen am RPG neu lanciert werden. 
Bevölkerung sorgte sich vor allem um die Erhaltung der offenen Landschaft nach Ausdehnung und Qualitäten.

Dieses Unterschwellige machte sich Luft mit einer bundesweit ergriffenen Verfassungsinitiative: „Schluss mit uferlosem Bau von Zweitwohnungen “, nicht von einer Partei oder einem landesweit agierenden Verband ergriffen, sondern von engagierten Privaten. Die Initiative wollte durch den Erlass eines Artikels in der Bundeverfassung (samt Übergangsbestimmung) den Anteil der Zweitwohnungen auf 20\% pro Gemeinde begrenzen, also die Zersiedlung mit einer absoluten Grenze stoppen. Eingereicht wurde sie am 18. Dezember 2007.20

Der Bundesrat empfahl - relativ zügig - die Ablehnung der Initiative. Er mahnte, sie entspreche als Bundesregelung nicht den unterschiedlichen föderativen Bedürfnissen in den einzelnen Kantonen. Er und das Parlament formulierten dennoch, aus Einsicht in die anstehende Problematik, novellierende Bestimmungen im Bundesgesetz über die Raumplanung (RPG) - gleichsam als (indirekter) Gegenvorschlag zur Initiative. Diese traten am 1. Juli 2011 in Kraft. ${ }^{21}$ Aber: Die Initianten zogen ihre Initiative nicht zurück, mit der Begründung, ihr eigener Vorschlag enthalte im Gegensatz zur vorgesehen abstrakten Regelung eine klare, einfache, ihrer Ansicht nach sogar maßvolle Lösung für ein „brennendes“ Problem.

Also kam es zu Abstimmung: Am 11. März 2012 wurde die Initiative durch Volk und Stände (Kantone) - für die breite Öffentlichkeit und die politischen Institutionen überraschend - angenommen, knapp zwar, aber eben mit den erforderlichen Mehrheiten. Die Verfassungsartikel traten mit der positiven Abstimmung gleich auch in Kraft. ${ }^{22}$

Auffallend, erst mit der Annahme wurde in der breiten Öffentlichkeit und bei den sog. Experten bewusst: Der genehmigte Verfassungsartikel 75 b BV über die Zweitwohnungen (samt einer Übergangsbestimmung) könnte weit mehr an offene Fragen in sich bergen als seit dem Einreichen der Initiative bei den meinungsbildenden Instanzen und in der Öffentlichkeit bewusst geworden sei. Die Anwendbarkeit sei fragwürdig, auch seien die wirtschaftlichen Folgen für viele Regionen beängstigend. Dies schlug relativ rasch in eine Negativstimmung um. Die Folgerung: Es gelte zwar das Abstimmungsergebnis zu respektieren, in erster Linie aber sei Rechtssicherheit durch Rechtsklarheit zu schaffen.

Bei genauerem Hinsehen fallen gleich mehrere Punkte auf. Einmal hatte wohl der Bundesrat die Ablehnung der Initiative empfohlen, nicht aber das Parlament. Das Bestehen eines indirekten Gegenvorschlages in Form der bereits in Kraft getretenen Revision des RPG kam sodann vor der Öffentlichkeit kaum zur Sprache. Der Abstimmungskampf, vor allem seitens der kritischen Parteien, wurde ohne jedes Engagement, ohne überlegene, kritische Sachkompetenz geführt. Den Gegnern der Vorlage ist es denn auch nicht gelungen ist, die sachliche, ökonomische und rechtliche Fragwürdigkeit des formulierten Verfassungsartikels bewusst zu machen. Das „unterschwellige“ Misstrauen und Unbehagen, resultierend aus den offenkundiger Versäumnisse seitens der Kantone, wurde völlig unterschätz, teilweise sogar übersehen oder gar als politisch nicht relevant abgetan.

Evident: Das durch die Initiative geschnürte politische Paket, auch in seiner vermeintlichen Einfachheit und Plausibilität, weckte keine politische Diskussion, rief nicht von sich aus nach Politikberatung. ${ }^{23}$ Wohl deshalb nicht, weil innert relativ kurzer Frist eine gesetzgeberische „Lösung“ im Rahmen des Bundesgesetzes übe die Raumplanung feststand. Regierung und Parlament vermeinten wohl, die Initiative werde zurückgezogen. Diese hielten sich denn auch eher zurück. Niemand mahnte, die landesweite Unterstützung aus dem Unterbewusstsein könnte dem Initiativbegehren zum Durchbruch verhelfen. Selbst das so typische Vernehmlassungsverfahren, das bei Referendumsvorlagen früh die Meinungsträger problemvertraut und hellhörig macht, wurde für einmal nicht mit Nachdruck mobilisiert. Selbst im Kontext der Revision des Bundesgesetzes über die Raumplanung - mit dem Einfügen von Aussagen zu den Zweitwohnungen - blieb die rechtliche/staatspolitische Problematik der Initiative eher zurückhaltend behandelt.

Darf vor diesem Hintergrund die nüchterne Feststellung gewagt werden, die Politikberatung, die an sich in der Demokratie betont hervortritt, habe im Umgang mit Initiativen, vor allem mit formulierten, den Ort und den Zeitpunkt ihrer Präsenznotwendigkeit nicht, resp. noch nicht gefunden, oder muss gar gefolgert werden, im Umgang mit Initiativen bleibe für sie zu wenig Aktionsraum? Wie dem auch sei, das begleitende Phänomen des unscharf erkennbar Unterschwellige hat die Politikberatung abgehalten, sich kritisch mit der Initiative auseinanderzusetzen. Sie muss dies aber lernen, weil aus Initiativen genauso geltendes (Verfassungs-)Recht werden kann wie aus dem ordentlichen Rechtssetzungsverfahren heraus.

\section{Grunderkenntnisse}

Die Politikberatung kann sich in der Schweiz nicht exklusiv als wissenschaftlich gestütztes/begründetes Einbringen von Sachkenntnissen und -erkenntnissen an die bekannten staatlichen Organe der Regierung und des Parlamentes verstehen. Sie lässt sich jedenfalls nicht darauf reduzieren.

In der Begegnung mit den politikrelevanten Instrumenten der direkten Demokratie - Referenden, Initiativen - wird es

20 Der Wortlaut der beantragte Teilrevision der BV (Art. 75 b sowie die Übergangsbestimmung Art. 197 Ziff. 8 BV) ist zentral für die Initiative, da es sich um eine formulierte Initiative handelt, siehe dazu http:// www.admin.ch/ch/d/as/2012/3627.pdf.

21 Siehe Art. 8 Abs. 2 und 3 RPG.

22 Die fragliche Bestimmung der formulierten Initiative ( 75 b BV, samt Übergangsbestimmung) wurde zum geltenden Verfassungsrecht mit Inkrafttreten am gleichen Datum der Annahme. Schwierig ist die Interpretation zu Fragen der unmittelbaren Anwendbarkeit und zum Problem, ob die konkretisierenden Bestimmungen auf dem Verordnungs- oder auf dem Gesetzesweg zu erlassen seien.

23 Das Problem der Zweitwohnungen galt offensichtlich nicht als politisch von hohem Gewicht. Selbst der dem Bundesamt beigegeben Rat für Raumordnung äußerte sich nicht mit Nachdruck vor der Öffentlichkeit. Es wäre an ihm gewesen, sich sachlich/rechtlich kritisch mit dem Initiativtext auseinanderzusetzen, allein schon um die eigenständige „Politikberatungskompetenz“ zu leben. 
für die Politikberatung unumgänglich, die mitlaufenden verwaltungs-, regierungs- und parlamentsseitigen Debatten um die Akzeptanz von Entscheidungen und Erlassen zu bedenken. Nicht im Sinne politischer Einflussnahme, aber - nach der unerlässlichen Klärung der offenen Sachfragen und der aufkommenden Probleme - in vorauseilender Auseinandersetzung mit den Argumenten des Pro und Contra bei den Volksabstimmungen aufgrund von Referenden und Initiativen.

Für die obligatorischen und fakultativen Referenden, insbesondere im Bereich der Verfassungs- und der (einfachen) Gesetzgebung versteht sich dieser „Zusatz“ der Politikberatung auch deshalb von selbst, weil die Abstimmungen zwingend durchzuführen sind resp. als (fakultative) vorbehaltene nur entfallen, wenn das Referendum nicht ergriffen wird. Sie sind in jedem Fall nicht konsultativ, sondern mit ihren Aussagen verbindlich. Die bürgernahe Demokratie mit ihren konstitutiven Volksabstimmungen zeitigt sodann aus sich heraus Vorwirkungen, die bei der Würdigung der Regelungsmöglichkeiten bedacht sein wollen. Die Politikberatung findet hier gleichsam einen besonderen Wirkungsraum vor.

Etwas heikler ist dieses Feld bei Volksinitiativen, weil diese in der Regel als formulierte eingereicht werden, was die politikberatende Auseinandersetzung auf Anträge zur Annahme oder Verwerfung resp. des Einbringens direkter oder indirekter Gegenvorschläge konzentriert. Aber auch diese Problemstellung vermittelt der Politikberatung über ihren Kernauftrag hinaus politiknahe Beratungsbereiche.

Mit den Hinweisen dürfte das nuanciert erweiterte Verständnis der Politikberatung für die Schweiz sichtbar geworden sein. Ergänzend zum Sachlichen/Zweckmäßigen fallen für sie die politischen/rechtlichen Randbedingungen der zu treffenden Regelungen in Betracht. Nochmals: Die Politikberatung darf sich ergänzende Reflexionen mit Blick auf die vorbehaltenen Volksabstimmungen zumuten, gleichsam zugunsten der politischen Rechte der Bürgerinnen und Bürger. Das Volk ist eben in der schweizerischen Demokratie in vielen Belangen potenzieller Entscheidungsträger. Die Akzeptierbarkeit von Erlassen und Vorhaben muss deshalb durch Verwaltung, Regierung und Parlament vorbedacht werden. Ein heikles, aber eine substanzielles Beratungsfeld, weil das Volk für die Politikberatung - im Gegensatz zu Verwaltung, Regierung und Parlament - nicht direkt ansprechbar ist.

\section{Literatur}

Giacometti, Z. (1994) [1985]. Echte und unechte Volksbefragung. In A. Kölz (Hrsg.), Zaccaria Giacometti, Ausgewählte Schriften (S. 59 ff). Zürich.

Häfelin, U., Haller, W., \& Keller, H. (2008). Schweizerisches Bundesstaatsrecht, 7. Aufl. Zürich: Schulthess.

Haller, Walter, (2009). The Swiss Constitution in a Comparative Context. Zürich/St.Gallen: Dike.

Jaag, T., Bucher, L., \& Häggi Furrer, R. (2011). Staatsrecht der Schweiz. Zürich/St. Gallen: Dike.

Lendi, M. (1997). Recht und Politik der Raumplanung, 2. Aufl. Zürich: vdf.

Lendi, M. (2004). Increasing Resistance to Political Consulting. In M. Koll-Schretzenmayr, M. Keiner \& G. Nussbaumer (Hrsg.), The Real and Virtual worlds of Spatial Planning (S. 127 ff). Berlin/Heidelberg: Springer. Lendi, M. (2005). Politikberatung. Nachfrage, Resonanz, Alibi. Zürich: vdf.

Lendi, M. (2006). Zur Geschichte der schweizerischen Raumplanung. DISP, 4/2006, $66 \mathrm{ff}$.

Lendi, M. (2008). Kommentar zu Art. 75 BV. In B. Ehrenzeller et al. (Hrsg.), Die Schweizerische Bundesverfassung - Kommentar, 2. Aufl. Zürich/St. Gallen: Dike/Schulthess.

Lendi, M. (2009). Das Unterschwellige - Potenzial politischer Kreativität oder politischer Verwerfungen. In M. Haslinger, A. Kanonier \& S. Zehetner (Hrsg.), Ein Jurist im Spannungsfeld von Wirtschaft, Technik und Recht, Festschrift für Franz Zehetner zum 60. Geburtstag (S. $421 \mathrm{ff})$. Wien: Neuer Wissenschaftlicher Verlag.

Lendi, M. (2012). Zweckmässiges, Gebotenes, Gerechtes - als Maßstäbe staatlichen Handelns. In M. Borchardt, T. Schrapel \& B. Vogel (Hrsg.), Was ist Gerechtigkeit? Befunde im vereinigten Deutschland. Köln: Böhlau.

Linder, W. (2008). Schweizerische Demokratie. Institutionen, Prozesse, Perspektiven, 3. Aufl. Bern: Haupt.

Neidhart L. (1970). Plebiszit und pluralitäre Demokratie, eine Analyse der Funktionen des schweizerischen Gesetzesreferendums. Bern: Francke.

Ruch, A. (2010). Umwelt - Boden - Raum. Basel: Helbing Lichtenhahn.

Steffen, I., \& Linder, W. (2006). Switzerland: Think Tanks and Vested Interests in Swiss Policy Making. German Policy Studies 3(2), 310-346.

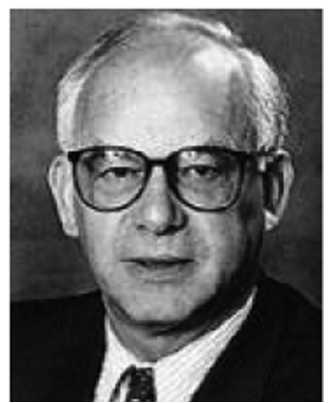

Prof. Dr. Martin Lendi ist Professor emeritus für Rechtswissenschaft an der Eidgenössischen Technischen Hochschule Zürich. Dort widmete er sich insbesondere der interdisziplinären Vernetzung der Rechtswissenschaft. E-Mail: martin.lendi@emeritus.ethz.ch. 